\title{
Stability of Subsonic Microjet Flows and Combustion*
}

\author{
Victor V. Kozlov ${ }^{1,2}$, Genrich R. Grek ${ }^{1}$, Mikchail M. Katasonov ${ }^{1}$, \\ Oleg P. Korobeinichev ${ }^{3}$, Yury A. Litvinenko ${ }^{1}$, Andrey G. Shmakov ${ }^{3}$ \\ ${ }^{1}$ Khristianovich Institute of Theoretical and Applied Mechanics, \\ Siberian Branch of Russian Academy of Sciences, Novosibirsk, Russia \\ ${ }^{2}$ Novosibirsk State University, Novosibirsk, Russia \\ ${ }^{3}$ Voevodsky Institute of Chemical Kinetics and Combustion Siberian Branch \\ of the Russian Academy of Sciences, Novosibirsk, Russia \\ Email: kozlov@itam.nsc.ru
}

Received July 18, 2013; revised August 27, 2013; accepted September 17, 2013

Copyright (C 2013 Victor V. Kozlov et al. This is an open access article distributed under the Creative Commons Attribution License, which permits unrestricted use, distribution, and reproduction in any medium, provided the original work is properly cited.

\begin{abstract}
Results of experimental studies of round and plane propane microjet combustion in a transverse acoustic field at small Reynolds numbers are presented in this paper. Features of flame evolution under the given conditions are shown. Based on the new information obtained on free microjet evolution, new phenomena in flame evolution in a transverse acoustic field with round and plane propane microjet combustion are discovered and explained.
\end{abstract}

Keywords: Round and Plane Jets; Acoustic; Flow Visualization; Combustion

\section{Introduction}

There are many papers on the study of premixed and/or non-premixed flames in association with acoustic forcing, for examples [1-4]. The experiments [5] gave an impetus to experimental investigations of the flame development during gas fuel combustion in microjets at low Reynolds numbers. The goal of the experiments [5] was to understand the mechanism of the flame bifurcation in a round jet generated by gas fuel combustion in a transverse acoustic field. However, this mechanism still remains unclear. The experimental studies [6-12] and other, where the influence of the initial conditions at the nozzle exit and acoustics on the development of round and plane microjets in the absence of combustion at low Reynolds numbers were investigated in detail, which made it possible to understand and explain some phenomena in the flame behaviour observed during propane combustion in these jets. The results of these studies are presented in this paper.

\section{Experimental Setup and Procedure}

The experiments were carried out using the experimental setup shown in Figure 1. A gas fuel (propane) or its mixture with air was fed into the nozzle through a honeycomb and a set of grids in the settling chamber. Four types of nozzles were used (Figure 1). The process of propane jet

\footnotetext{
*Short paper.
}

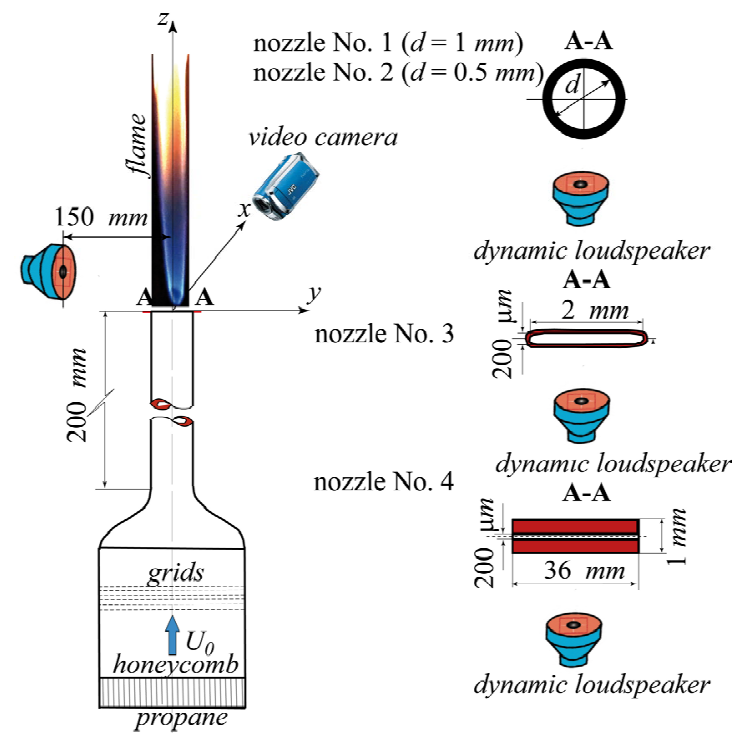

Figure 1. Experimental set-up.

combustion subjected to the influence of a transverse acoustic field was registered by a digital video camera. The transverse acoustic field was created with the help of a dynamic loudspeaker excited by sinusoidal electric signals of different frequencies (from 1 to $7.5 \mathrm{kHz}$ ). Intensity of sound from the loudspeaker was about 90 decibels.

The jet velocity was measured by a precision flow meter. The range of jet velocities investigated was between 
12 and $30 \mathrm{~m} / \mathrm{sec}$.

\section{Results and Discussions}

\subsection{Diffusion Combustion of Propane Round Microjet}

The flame of round mini and microjets are transformed into plane microjet through flattening process in a transverse acoustic field (Figure 2). In this situation, the jet as a whole performs sinusoidal oscillations.

The sinusoidal oscillations of the jet result in its bifurcation into two jets far downstream (Figures 3 and 4). It should be noted that the frequency of the transverse acoustic field is approximately doubled as the nozzle exit diameter is reduced from 1 to $0.5 \mathrm{~mm}$.

The flame behaviour is correlated with the process of round free jet flattening and bifurcation in a transverse acoustic field [6]. Acoustic forcing of a slit burner flame was experimentally investigated in [5], and it was confirmed that the bifurcation of the jet due to acoustic forcing is basically a two-dimensional phenomenon. Thus, the bifurcation mechanism of the round mini- or microjet flame in a transverse acoustic field is related to free jet flattening or, in other words, round jet transformation to a plane (2D) jet. The sinusoidal oscillations of the free plane jet result in its splitting into two jets. The flame propagates over the external surface of the jet.

\subsection{Diffusion Combustion of a Plane Propane Microjet}

As the jet velocity increases, the flame sharply lifts-off from the nozzle at a certain threshold value of the jet

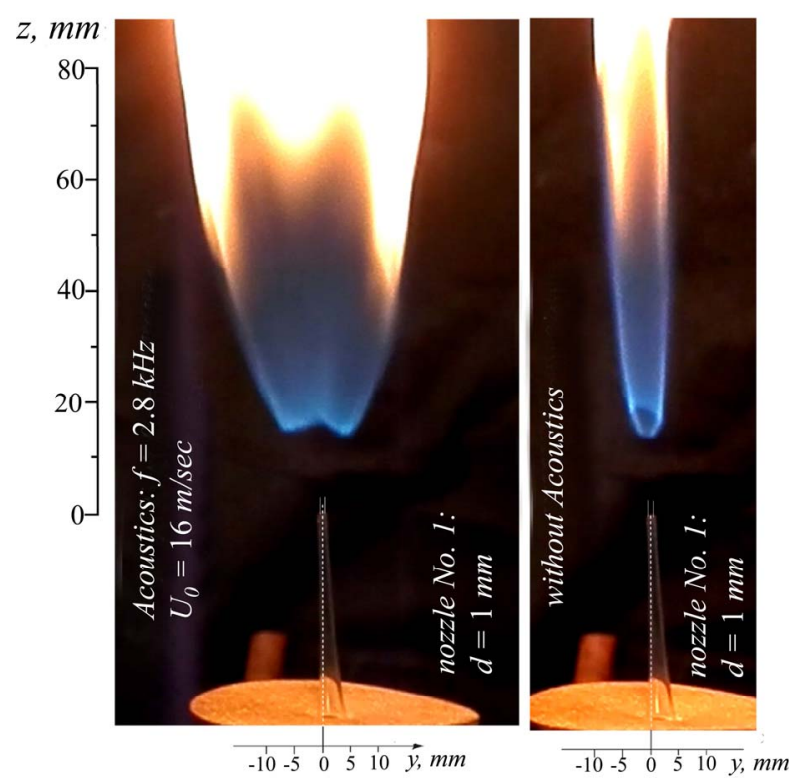

Figure 2. Round microjet flame flattening under the action of a transverse acoustic field.
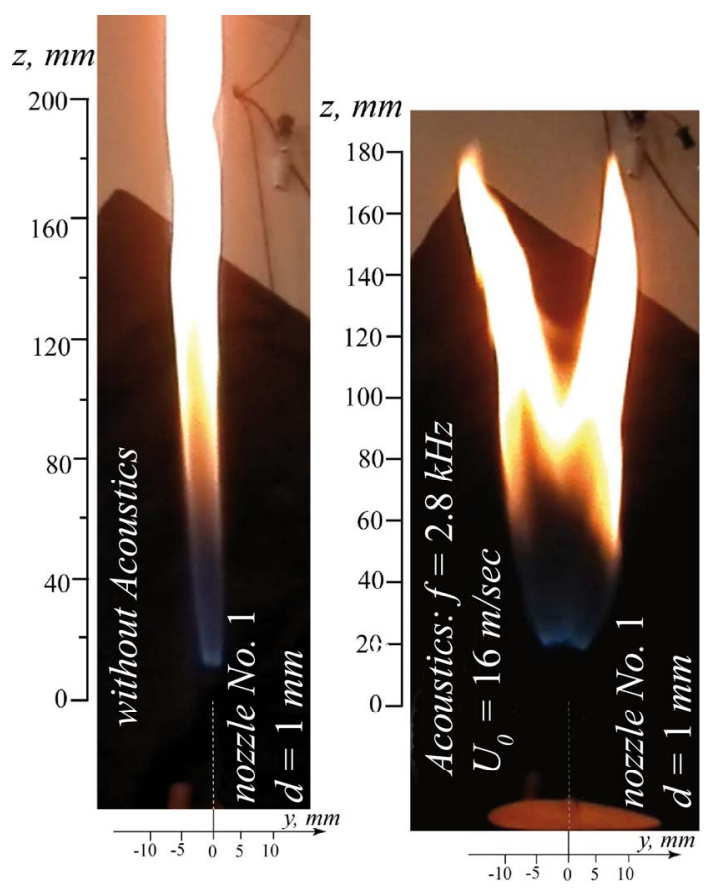

Figure 3. Round microjet (for nozzle $d=1 \mathrm{~mm}$ ) flame bifurcation in a transverse acoustic field.
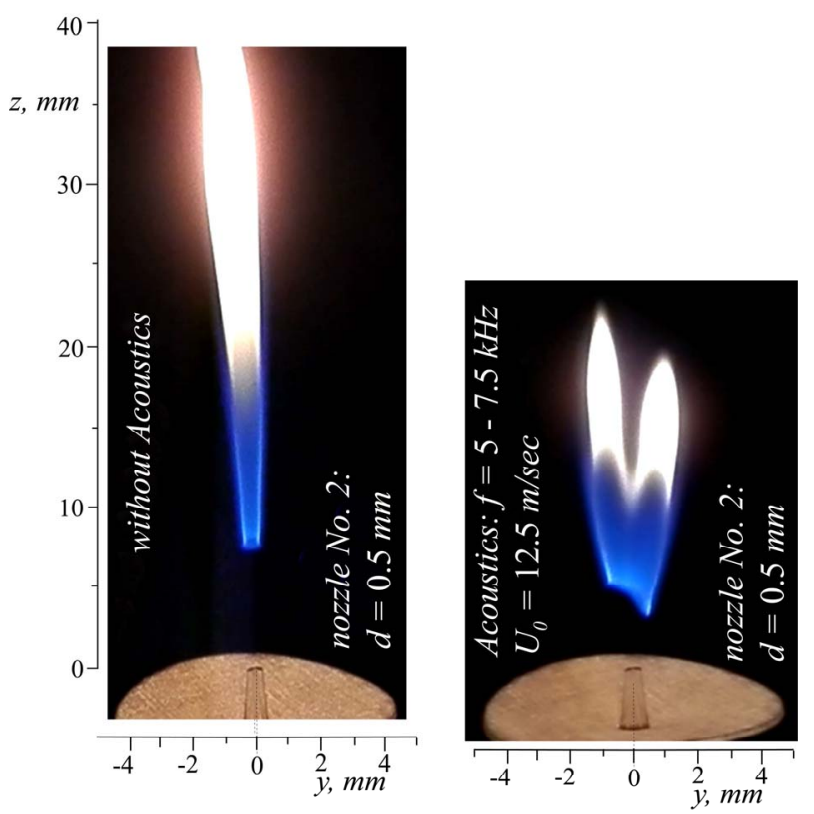

Figure 4. Round microjet (for nozzle $d=0.5 \mathrm{~mm}$ ) flame bifurcation in a transverse acoustic field.

velocity, and the so-called lifted flame is formed (Figure 5). In this situation, a flame bifurcation under the acoustic influence is clearly observed in the frequency range from 1 to $3 \mathrm{kHz}$ (Figure 5). Apparently, acoustics stabilizes the combustion process and promotes intensification of heat/mass transfer between propane and air, which results in combustion region spreading and, finally, in the flame bifurcation. 


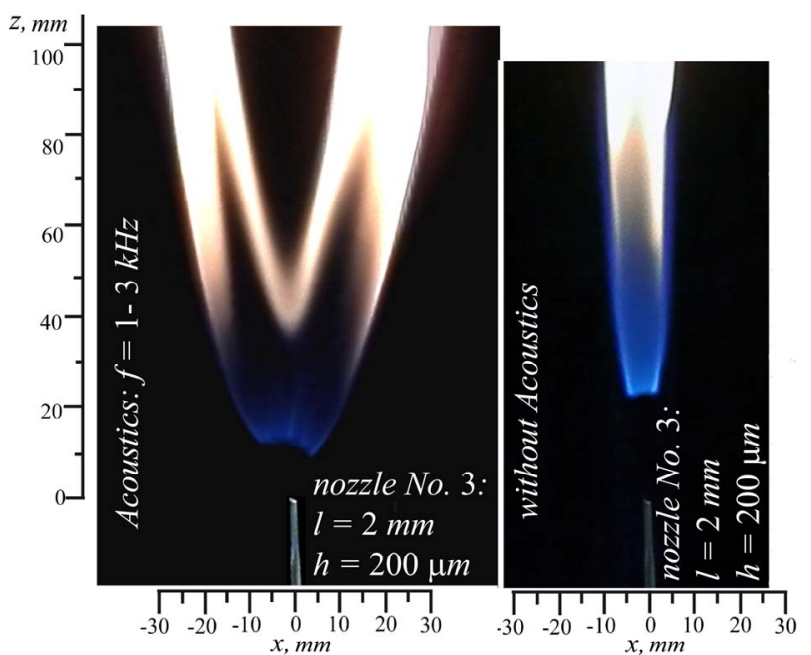

Figure 5. Plane microjet flame bifurcation in a transverse acoustic field.

The flame evolution of a plane microjet in a transverse acoustic field in the yz-plane is shown in Figure 6.

In this case, in contrast to the short nozzle situation, it is possible to observe not only the process of the flame bifurcation in a transverse acoustic field, but also flame folding due to the edge effect. This folding occurs in directions opposite to each other on each period of the acoustic effect (Figures 6(b) and (c)). In the course of diffusion combustion of this propane jet, it is also possible to observe flame propagation on the surfaces of these two bifurcated and rolled-up jets (Figure 6(a)).

The flame pattern of the jet without acoustics and with acoustics (Figure 7(a)) and also the smoke visualization pattern of this jet in the absence of combustion (Figure 7(b)) illustrate the jet flame bifurcation process under the influence of a transverse acoustic field. The bifurcation and broadening both of the jet and its flame in the xzplane can be seen. The flame broadening region is approximately twice greater than that in the case with no acoustic influence (Figure 7(a)).

\subsection{Combustion of a Plane Microjet of a Propane-Air Mixture}

The flame development during premixed propane-air mixture combustion [13] in a plane microjet under the action of a transverse acoustic field was experimentally studied. The propane-air ratio was $37.2 \% / 62.8 \%$, respectively (the equivalence ratio was $\varphi=14$ ). At smaller equivalence ratios, the transverse acoustic field produced a much weaker effect on jet combustion, if any at all. As in the situation of diffusion combustion of a plane propane microjet (Figure 5), the plane microjet flame in the case of combustion of a premixed propane-air mixture also experienced a bifurcation under an acoustic effect (Figure 8). The jet flame in the absence of the acoustic field and under

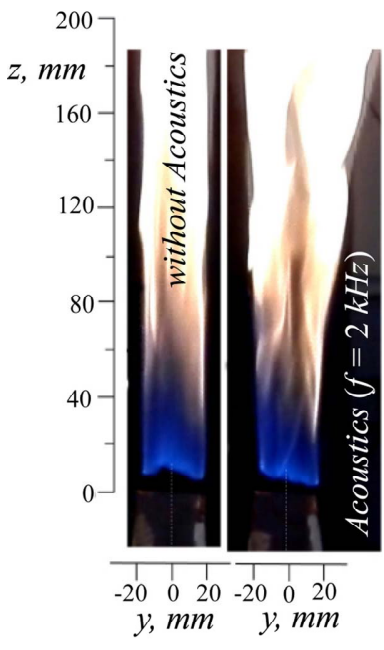

(a)

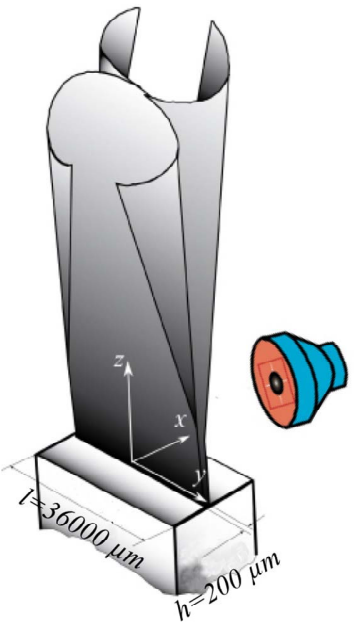

(b)

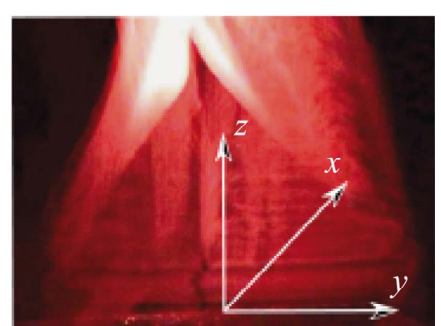

(c)

Figure 6. Plane microjet flame bifurcation in a transverse acoustic field: (a) pattern of flame folding, (b) scheme of the free jet bifurcation and folding, (c) smoke visualization pattern of free jet folding (taken from [11]).

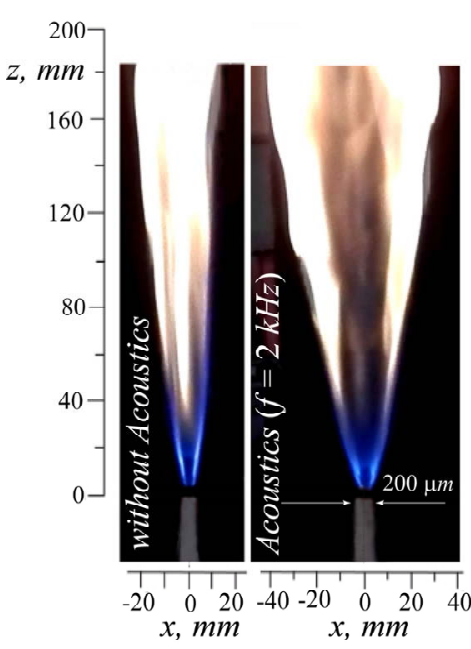

(a)

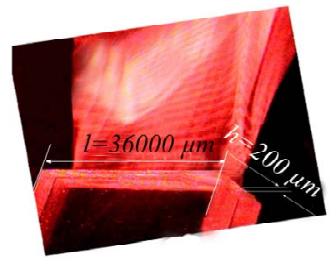

(b)
Figure 7. Plane microjet flame bifurcation in a transverse acoustic field: (a) pattern of the flame bifurcation, (b) smoke visualization pattern of the jet bifurcation without combustion (taken from [11]).

the acoustic effect at frequencies smaller than $500 \mathrm{~Hz}$ is attached to the nozzle.

It is known that the lift-off of the flame from the noz- 


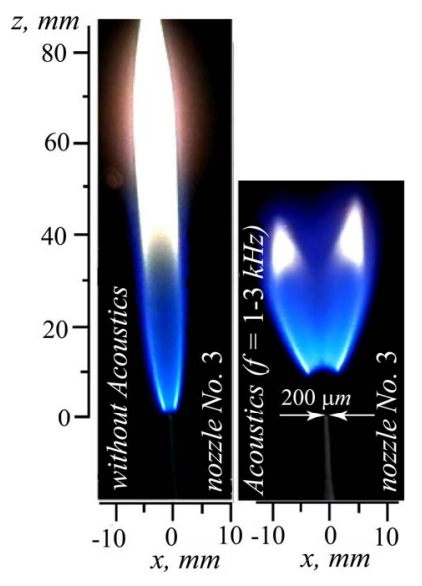

Figure 8. Plane microjet flame bifurcation in a transverse acoustic field: short nozzle $(I=2 \mathrm{~mm}, h=200 \mu \mathrm{m}, l / h=10)$.

zle exit occurs as the jet velocity increases or, as in this case, when the jet velocity remains constant; flame separation from the nozzle exit occurs owing to the acoustic influence at certain frequencies $(f=1-3 \mathrm{kHz})$. In the situation considered here (Figure 8), the lifted flame is sharply broadening in the $x z$ plane, and its bifurcation can be observed further downstream, as well as in the previous case of diffusion combustion of the plane microjet subjected to an acoustic effect (Figure 5).

\section{Conclusion}

On the basis of the information obtained on the evolution of microjet flows, some new phenomena in the behaviour of the flame during microjet combustion in a transverse acoustic field have been discovered and explained for the first time. It has been shown for the first time that the flame during round propane microjet combustion displays flattening and bifurcation; the behaviour of the jet in the absence of combustion has been also studied. A similar result has been found for the first time in the flame behaviour during diffusion and premixed combustion of propane microjets in a transverse acoustic field, that is, flame bifurcation and folding, as in the case of the jet development in the absence of combustion.

\section{Acknowledgements}

This work was supported by the Integration Project SB RAS No. 24 and by the Russian Foundation for Basic Research, project Nos. 11-01-00292, 12-08-31083, 1207-00548, and 13-07-00616.

\section{REFERENCES}

[1] F. Baillot and D. Demare, "Physical Mechanisms of a Lifted Nonpremixed Flame Stabilized in an Acoustic Field," Combustion Science and Technology, Vol. 174, No. 8, 2002, pp. 73-98.

\section{http://dx.doi.org/10.1080/713713066}

[2] W. B. Ng, A. F. Salem and Y. Zhang, "Three-Dimensional Visualization of Diffusion Flame Shapes under Acoustic Excitation Using Stereoscopic Imaging and Reconstruction Technique,” Journal of Visualization, Vol. 6, No. 4, 2003, pp. 329-336. http://dx.doi.org/10.1007/BF03181739

[3] M. Suzuki, T. Atarashi and W. Masuda, "Behavior and Structure of Internal Fuel-Jet in Diffusion Flame under Transverse Acoustic Excitation," Combustion Science and Technology, Vol. 179, No. 12, 2007, pp. 2581-2597. http://dx.doi.org/10.1080/00102200701487012

[4] Q. Wang, Y. Zhang, H. J. Tang and M. Zhu, "Visualization of Diffusion Flame/Vortex Structure and Dynamics under Acoustic Excitation," Combustion Science and Technology, Vol. 184, No. 10-11, 2012, pp. 1445-1455. http://dx.doi.org/10.1080/00102202.2012.693419

[5] M. Suzuki, S. Ikura and W. Masuda, "Comparison between Acoustically-Excited Diffusion Flames of Tube and Slit Burners," 11th Asian Symposium on Visualization, Niigata, 2011, pp. 1-6.

[6] V. V. Kozlov, G. R. Grek, A. V. Dovgal and Y. A. Litvinenko, "Stability of Subsonic Jet Flows,” JFCMV 9, 2013 (accepted paper).

[7] V. V. Kozlov, G. R. Grek, G. V. Kozlov and M. V. Litvinenko, "Visualization of the Processes of Evolution and Turbulent Breakdown of a Low-Speed Round Jet," Visualization of Mechanical Processes, Vol. 1, No. 2, 2011, pp. 1-18.

[8] G. V. Kozlov, G. R. Grek, A. M. Sorokin and Y. A. Litvinenko, "Initial Conditions Effect at the Nozzle Exit on the Round Jet Structure," Thermophysics and Aeromechanics, Vol. 15, No. 1, 2008, pp. 59-68. http://dx.doi.org/10.1134/S0869864308010046

[9] V. V. Kozlov, G. R. Grek, Y. A. Litvinenko, G. V. Kozlov and M. V. Litvinenko, "Influence of Initial Conditions at the Nozzle Exit and Acoustical Action on the Structure and Stability of a Plane Jet," Visualization of Mechanical Processes, Vol. 2, No. 3, 2012, pp. 1-29.

[10] G. R. Grek, G. V. Kozlov, V. V. Kozlov, Y. A. Litvinenko and M. V. Litvinenko, "Round Jet in Cross Shear Flow," Visualization of Mechanical Processes, Vol. 2, No. 4, 2012, pp. 1-29.

[11] V. V. Kozlov, G. R. Grek, Y. A. Litvinenko, G. V. Kozlov and M. V. Litvinenko, "Round and Plane Jets in a Transverse Acoustic Field," Journal of Engineering Thermophysics, Vol. 20, No. 3, 2011, pp. 272-289. http://dx.doi.org/10.1134/S1810232811030052

[12] Y. A. Litvinenko, G. R. Grek, V. V. Kozlov and G. V. Kozlov, "Subsonic Round and Plane Macrojets and Microiets in a Transverse Acoustic Field," Doklady Physics, Vol. 56, No. 1, 2011, pp. 26-31. http://dx.doi.org/10.1134/S1028335811010046

[13] J. Warnatz, U. Maas and R. W. Dibble, “Combustion Physical and Chemical Fundamentals, Modeling and Simulations, Experiments, Pollutant Formation,” Springer, Berlin, 2001, pp. 1-350. 